\title{
Predicting prognosis of BRAF mutated melanoma due to analyzing immune response
}

Nakamura Kenta, Ashida Atsuko, Kiniwa Yukiko, Okuyama Ryuhei

Department of Dermatology, Shinshu University School of Medicine

3-1-1 Asahi, Matsumoto, Nagano 390-8621, Japan

\section{Background}

Melanocarcinoma is a highly malignant form of skin cancer. Recently, the relationship between melanocarcinoma prognosis and immune reactions has been garnering interest. The high therapeutic effects of immunity checkpoint inhibitors have been reported in cases in which numerous CD8-positive t-cells have infiltrated into the tumor. Daud Al, et al : J Clin Invest : 2016

Furthermore, melanocarcinoma has many genetic mutations, of which BRAF-mutant is the most common, being observed in approximately $30 \%$ of Japanese patients. The MAPK pathway and PI3 downstream are activated excessively by the BRAF-mutant to increase cell proliferation. Moreover, immunosuppressant cytokines are produced by cancer cells, and inhibits the immune response to melanocarcinoma. The use of BRAF inhibitors became possible for BRAF-mutant melanocarcinoma since last year. Although this treatment is more effective than the conventional chemotherapy drug dacarbazine, the cancer develops tolerance to the drug around 7 months into treatment, which can cause it to resist the treatment and grow. Sumimoto $\mathrm{H}$, et al : J Exp Med, 203 : 2006

As a method to solve the problems related to tolerance of BRAF inhibitors, a therapy involving the concomitant use of drugs with different mechanisms of action is now gaining attention. Recently, the combined therapy with molecularly targeted drugs (MEK inhibitors) that act on the downstream signals of BRAF has been approved, and clinical testing for combined use with immunity checkpoint inhibitors is also being studied in clinical trials overseas.

There are no Japanese single-center studies on BRAF--mutant melanocarcinoma reports that have investigated the relationship between immunity responses before treatment and long-term prognosis in Japan. We therefore conducted this investigation to provide basic data for new immunotherapies such as immunity checkpoint inhibitors of which we can expect the popularity to rise in the future.

\section{Aim}

Predicting prognosis of BRAF mutated melanoma due to analyzing immune response

\section{Subject and Method}

We analyzed 30 cases in which the primary tumor was a BRAF-mutant positive melanocarcinoma (stages II and III) who were treated at the Dermatology department of the Shinshu University School of Medicine in the 15-year period between 2000 and 2014 . The mean age of patients at initial consultation was 57.6 years (19-87 years) and the median age was 60 years. As 14 were male and 16 were female, the sex ratio was approximately equal. In terms of anatomical categories, 20 cases

(approximately 66.6\%) were located in the hairy parts, trunk or four limbs, 5 cases $(16.6 \%)$ were located in the head and neck region (excluding hairy portions) and 5 cases (16.6\%) in the hands or feet. We performed

immunostaining of the resected tumor specimen (CD8, GranzymeB) and captured images with (NanoZoomer, Hamamatsu Photonics, Hamamatsu city, Japan), randomly selected 10 visual fields of the tumor site and counted with ImageJ to calculate the mean.

\section{Result}

The cut-off value was set at $250 / \mathrm{mm} 2$ for CD8 and $150 / \mathrm{mm} 2$ for Granzyme B. Cases that were categorized in the high group for both CD8 and Granzyme B were categorized as the high group, while all other cases were categorized as the low group.

Figure 1

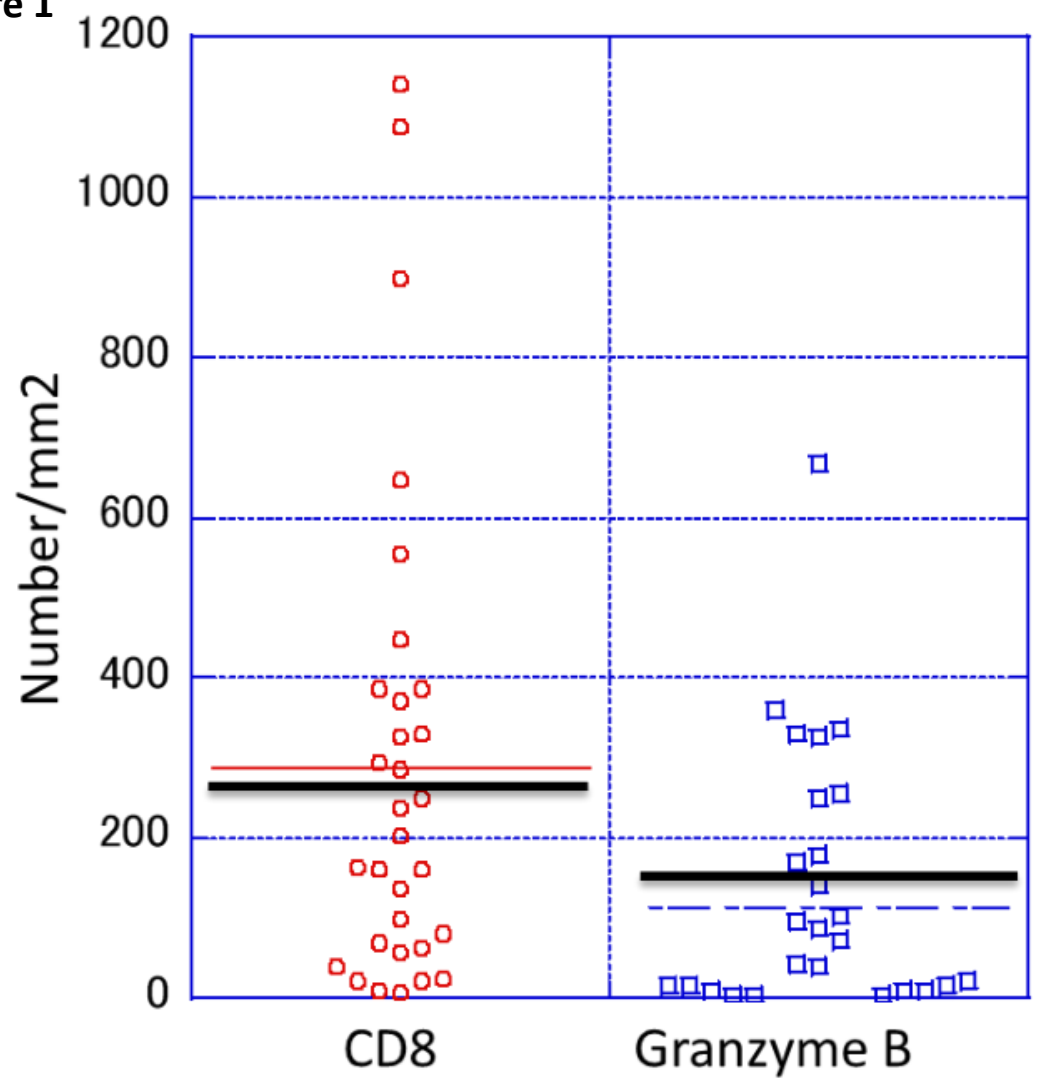

High

Cut-off value

There was no significant difference in the disease duration between the high and low groups of CD8, Granzyme B and combination groups, respectively (according to AJCC2009 version's TNM categorization)
We did not find a significant difference between the respective disease specific survival rates and recurrence-free survival rates of the high and low groups for CD8 and Granzyme B.

Figure 2 CD8

$$
\text { Disease-specific survival rate }
$$
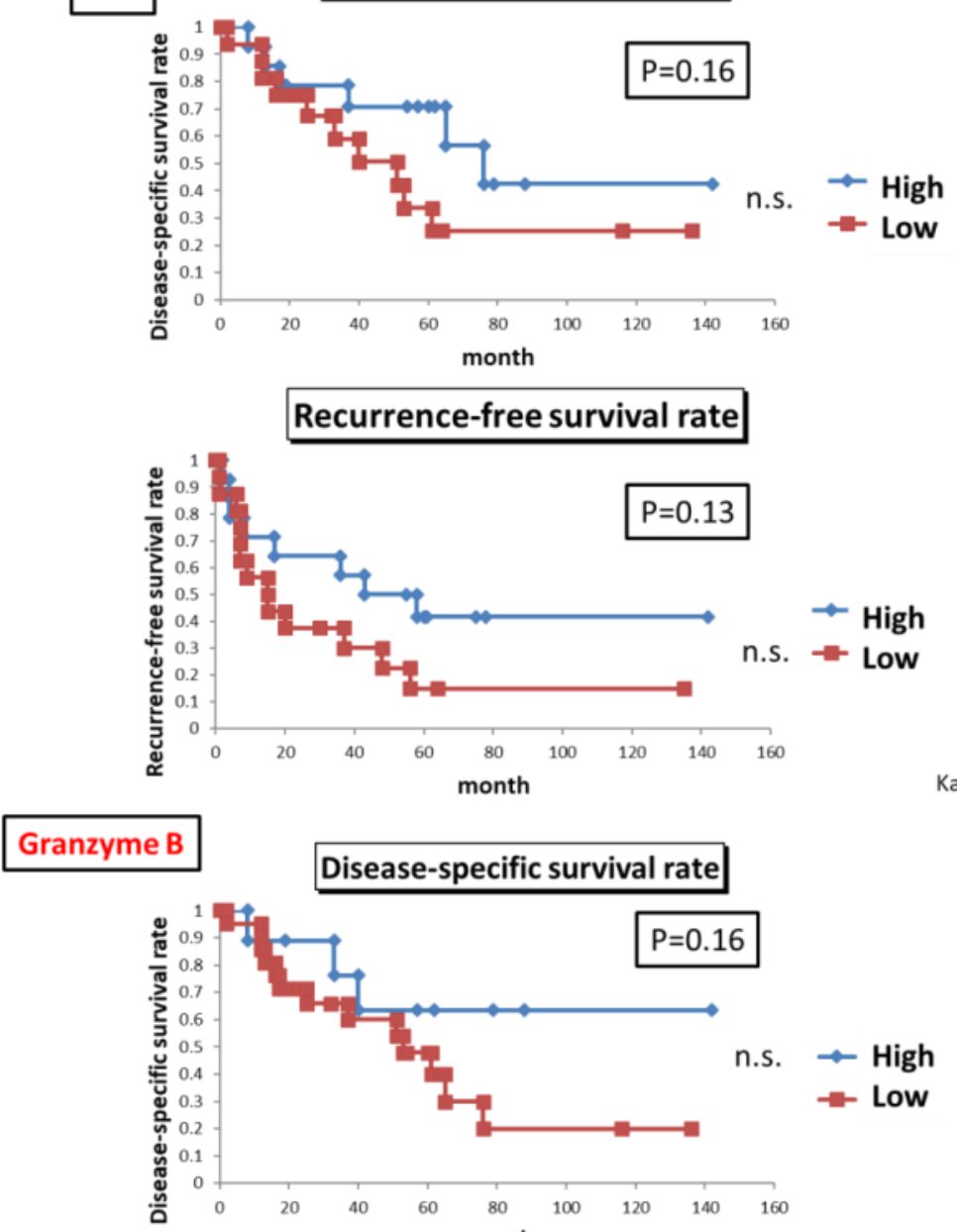

Recurrence-free survival rate

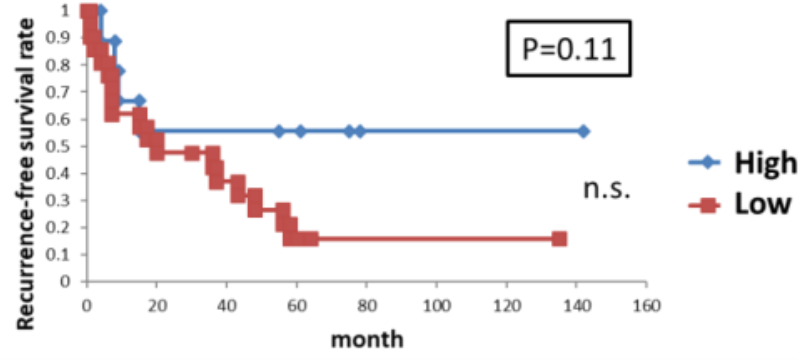

The disease-specific survival and recurrence-free survival rates were significantly higher in the high group than the low group for both CD8 and Granzyme B $(p=0.03)$.

CD8 and Granzyme B

Figure 3

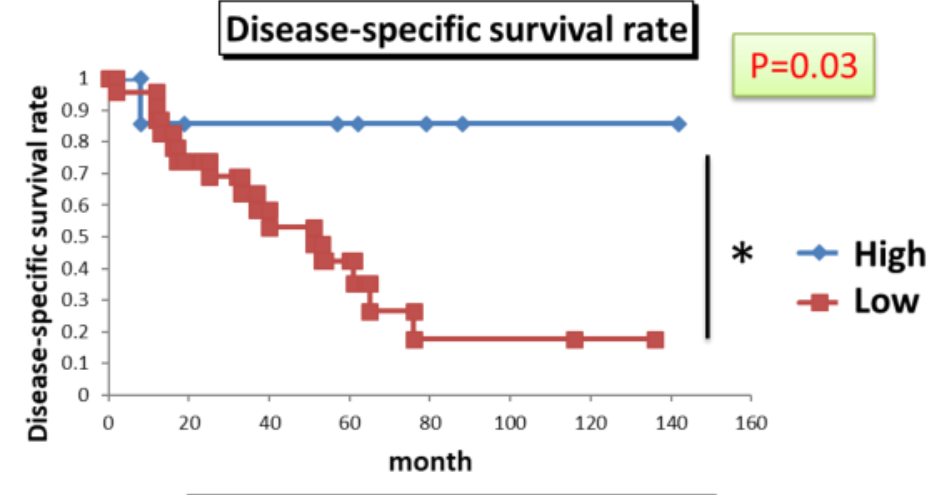

Recurrence-free survival rate

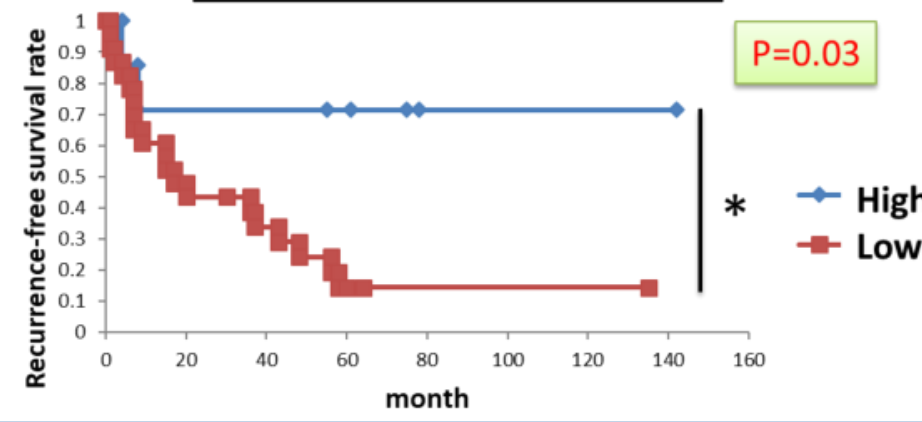

\section{Conclusion and Discussion}

Based on the above, it appeared that in cases in which the CD8 or Granzyme B was low according to immunostaining of the resected tumor specimen, the post-operative recurrence rate was high. Furthermore, as the disease-specific survival rate was low, such cases need to undergo postoperative adjuvant therapy with careful observation of subsequent progress. As tumor-infiltrating CD8-positive t-cells emit Granzyme B to eliminate the tumor, past studies have also reported on the relationship between the expression of tumor-infiltrating CD8-positive t-cells or Granzyme B with prognosis and recurrence. However, there have very few reports of this nature in Japan to date. Also, since there are no reports on analyses focusing on BRAF-mutant positive cases, the information in the present study could be useful for developing future treatment strategies. More data will be gathered on similar cases and the relationship between the efficacy of BRAF inhibitors and CD8 and Granzyme B values of the resected tumor specimens will be analyzed going forward. 